\title{
22-km distributed temperature sensor using Brillouin gain in an optical fiber
}

\author{
X. Bao, D. J. Webb, and D. A. Jackson \\ Applied Optics Group, Physics Laboratory, The University, Canterbury, Kent CT2 7NR, UK
}

Received December 15, 1992

\begin{abstract}
We describe an experimental distributed temperature sensor that uses the temperature dependence of the Brillouin frequency shift. When a $22.2-\mathrm{km}$ sensing length is used, we have observed a temperature resolution of $1^{\circ} \mathrm{C}$ and have obtained a spatial resolution of $10 \mathrm{~m}$.
\end{abstract}

During the past ten years, several different schemes have been demonstrated for realizing a distributed temperature sensor (DTS) with an optical fiber as the sensing element. The evolution of the state of polarization along the fiber, ${ }^{1}$ the temperature dependences of the Rayleigh backscattering coefficient, ${ }^{2}$ and the ratio of Stokes to anti-Stokes intensities for Raman scattering ${ }^{3}$ have all been used as the sensing mechanism.

More recently, the variation with temperature of the Stokes frequency shift in stimulated Brillouin scattering has been proposed as a suitable mechanism for a DTS. ${ }^{4}$ A related technique is based on the variation with temperature of the center frequency of the Brillouin gain curve. This approach has been demonstrated by Kurashima et $a l^{5}{ }^{5}$ They reported a temperature resolution of $3{ }^{\circ} \mathrm{C}$ combined with a spatial resolution of $100 \mathrm{~m}$ over $1.2 \mathrm{~km}$ of fiber for their approach, which they describe as Brillouin optical-fiber time-domain analysis (BOTDA). In this Letter we describe a DTS system based on BOTDA that has demonstrated a temperature resolution of $1^{\circ} \mathrm{C}$, a spatial resolution of $10 \mathrm{~m}$, and a sensing length of more than $22 \mathrm{~km}$.

The technique operates as follows: Light from a $\mathrm{cw}$ probe laser is launched into one end of the fiber while short pulses of light from a pump laser are launched into the other end. When the pump laser frequency is greater than that of the probe laser by the Brillouin frequency shift, the probe laser will be amplified, experiencing Brillouin gain. ${ }^{6}$ The Brillouin frequency shift depends on the fiber temperature; so, when the fiber temperature is nonuniform, the probe will experience gain for a given laser frequency difference only in those parts of the fiber at one particular temperature.

If the probe intensity emerging from the fiber is monitored following the launch of a pump pulse, an increase in the intensity will be observed whenever Brillouin gain occurs. The time delays between the launch of the pump pulse and these increases in the received probe signal correspond to round-trip times for light traveling to and from the regions of gain. These times provide the positional information. If the laser frequency difference is adjusted, then the probe will experience gain in parts of the fiber at a different temperature. Therefore, by slowly scanning one of the laser frequencies we can map the temperature distribution of the whole fiber.

The experimental arrangement is illustrated in Fig. 1. Both lasers were solid-state cw diodepumped Nd:YAG lasers emitting close to $1319 \mathrm{~nm}$. The launched power of the probe laser was approximately $7 \mathrm{~mW}$. The optical frequency of the probe laser could be adjusted by temperature tuning the cavity. A Bragg cell was used as an optical switch to provide 100-1000-ns pulses from the pump laser, and the peak launched pump power was approximately $5 \mathrm{~mW}$ from the first-order diffracted beam, which was monitored at photodetector D1. The zeroth-order beam was mixed with light from the probe laser by directional coupler DC1 and the beat frequency monitored by a fast $(20-\mathrm{GHz})$ detector and a microwave spectrum analyzer.

Most of the power in the launched pump pulses was directed into one end of the $22-\mathrm{km}$ sensing fiber by a $90: 10$ coupler, DC2, while the probe light was directed into the other end through a 60:40 coupler, DC3. The probe light emerging from the sensing fiber was monitored on a storage oscilloscope by a p-i-n diode detector, D2, and an amplifier with a combined bandwidth of more than $50 \mathrm{MHz}$. Most of the sensing fiber was at ambient temperature. However, two 500-m lengths of the fiber were placed in temperature-controlled ovens. The first length began $10.6 \mathrm{~km}$ from the pump input end of the fiber and the second length $17.4 \mathrm{~km}$ from the same end. There were six splices over the whole fiber length.

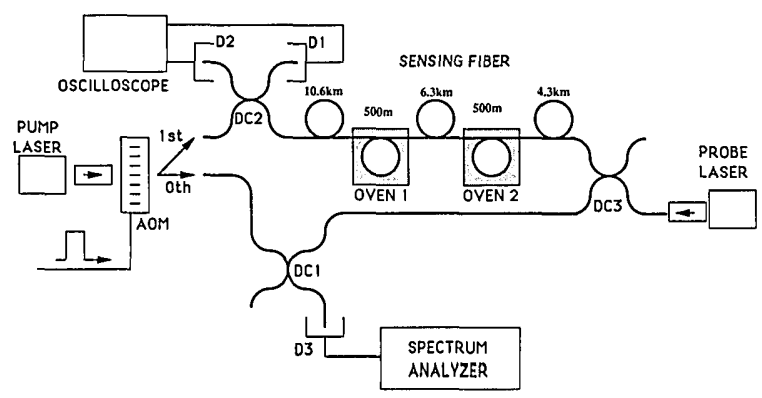

Fig. 1. Experimental arrangement: D1-D3, photodetectors; AOM, acousto-optic modulator; DC1-DC3, directional couplers. 


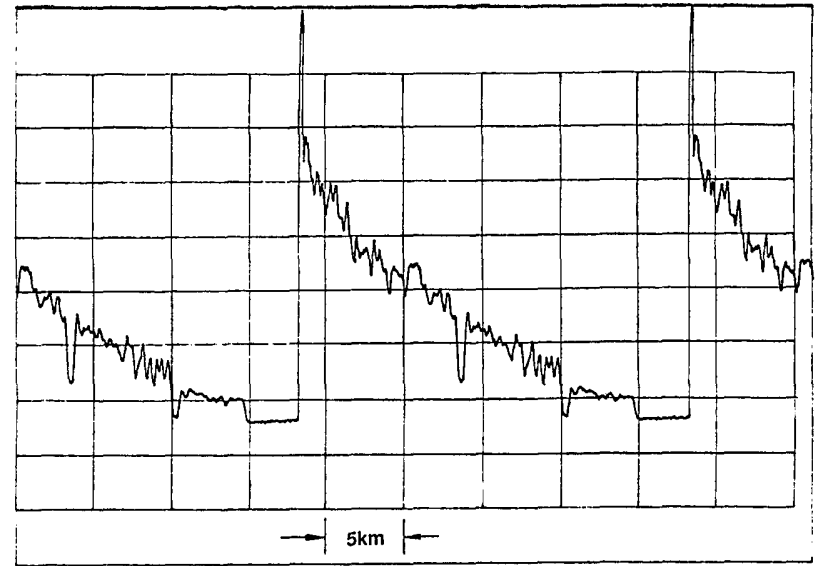

(a)

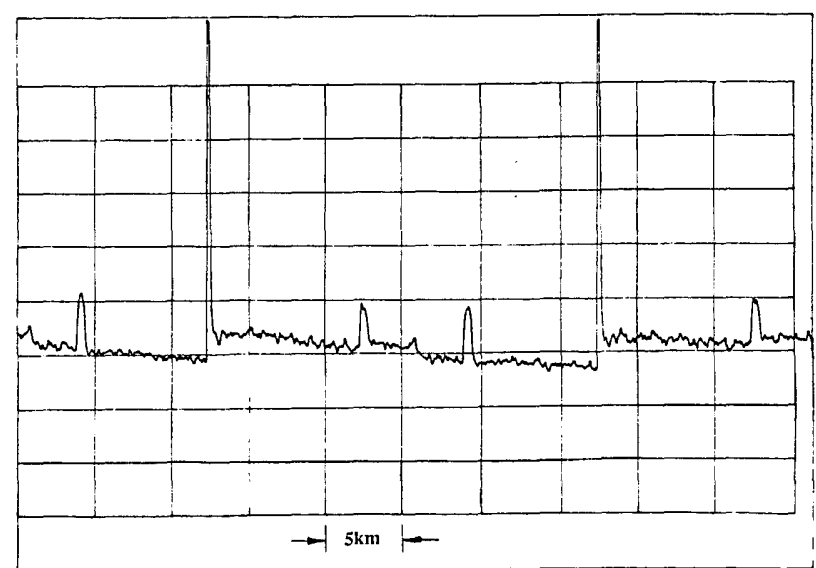

(b)

Fig. 2. Oscilloscope traces showing probe intensity monitored at D2 (Fig. 1): (a) probe frequency optimized for fiber at ambient temperature, (b) probe frequency optimized for fiber at $33^{\circ} \mathrm{C}$. The time base is $50 \mu \mathrm{s} /$ division, which corresponds to $5 \cdot \mathrm{km}$ fiber length per division, and the vertical scale is in arbitrary units.

All optical launching and delaunching was carried out with $20 \times$ objectives, and manual polarization controllers were inserted into the system at the launch of the probe and first-order diffracted pump light. We found that the Brillouin gain could be maximized over the whole fiber length by adjustment of the polarization states. However, the gain process could never be completely destroyed.

Figure 2 shows sample traces obtained from the storage oscilloscope monitoring the probe-beam intensity after the launch of a pump pulse of 200-ns width, corresponding to a $20-\mathrm{m}$ spatial resolution. In Fig. 2(a) the probe frequency was set to optimize the Brillouin gain for the fiber at ambient temperature. In Fig. 2(b) the probe frequency was adjusted to optimize the Brillouin gain for the fiber in the second oven at $33^{\circ} \mathrm{C}$. The first oven temperature was $30^{\circ} \mathrm{C}$, and a clear signal from the fiber in this oven may also be seen. Figure 3 is a plot of the laser beat frequency corresponding to the maximum Brillouin gain that occurs in the first oven as a function of its temperature. The pump pulse width used was $500 \mathrm{~ns}$, corresponding to a $50-\mathrm{m}$ spatial resolution.
For these data, the root-mean-square deviation from the best straight line was $0.8^{\circ} \mathrm{C}$ and the temperature coefficient of the Brillouin frequency shift was determined to be $1.3 \pm 0.1 \mathrm{MHz} /{ }^{\circ} \mathrm{C}$. Other data taken for both ovens demonstrated deviations from linearity of between 0.7 and $1^{\circ} \mathrm{C}$; hence we may set a lower limit on the temperature resolution of this system of $1^{\circ} \mathrm{C}$.

The temperature resolution of the system is further illustrated in Fig. 4, which shows plots of the probebeam intensity (as viewed on the oscilloscope) as a function of the laser beat frequency for six different temperatures at a point in the fiber in the center of oven 1. The shift in the peak of the Brillouin gain curve with temperature is clearly visible.

To demonstrate the spatial resolution of the system, we reduced the duration of the probe pulses to $100 \mathrm{~ns}$ and adjusted the laser beat frequency to optimize the Brillouin gain in the first oven, which

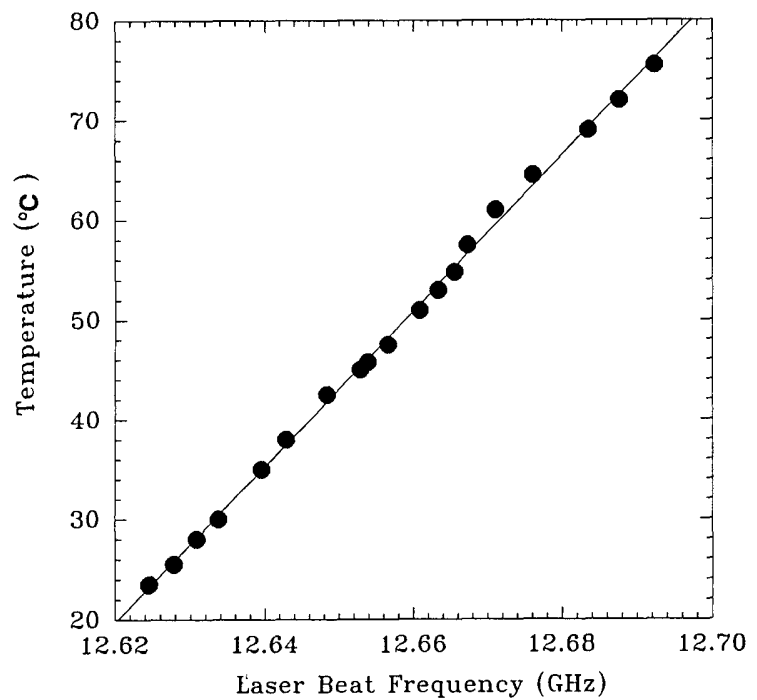

Fig. 3. Laser beat frequency giving maximum Brillouin gain for the fiber in oven 2 as a function of oven temperature.

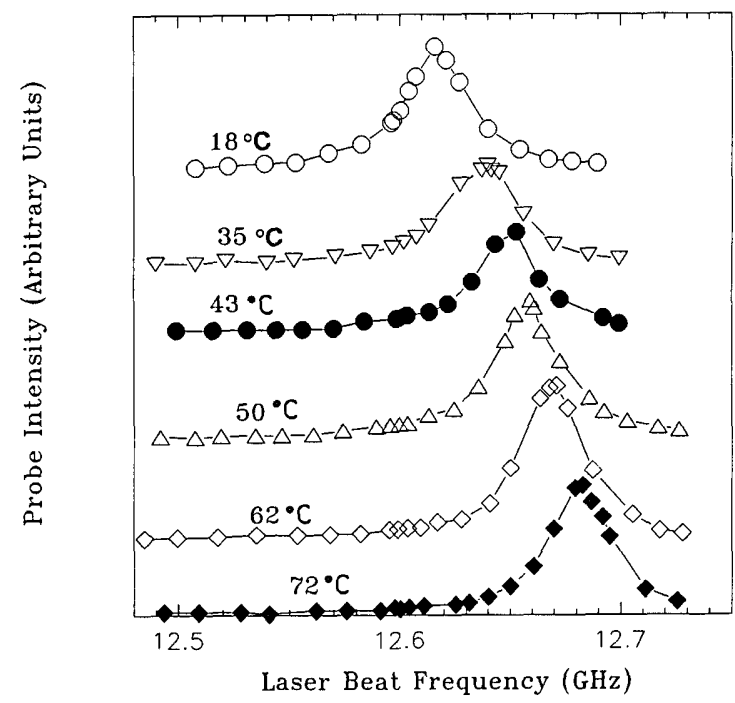

Fig. 4. Plot of probe-beam intensity as a function of laser beat frequency for a point in the center of oven 1 at six different temperatures. 


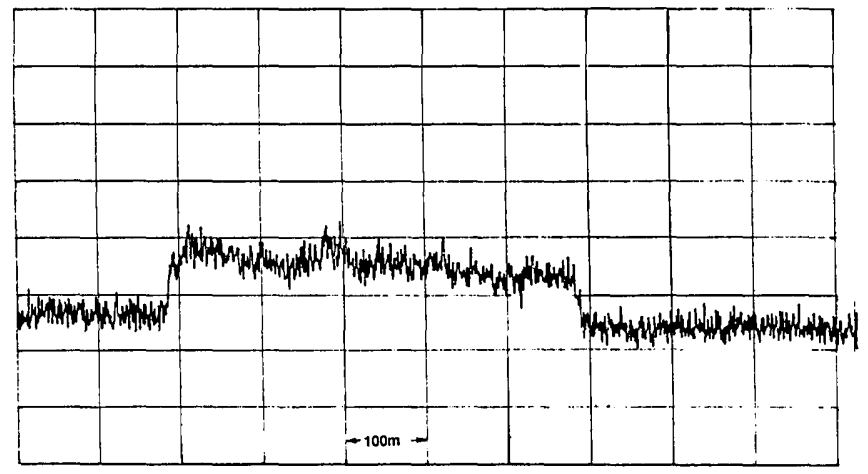

Fig. 5. Expanded view of an oscilloscope trace of probe intensity as a function of time for a section of fiber surrounding oven 1 (enhanced signal) at $30^{\circ} \mathrm{C}$. The time base is $1 \mu \mathrm{s} /$ division, which corresponds to $100-\mathrm{m}$ fiber length per division.

was at a temperature of $30^{\circ} \mathrm{C}$. Figure 5 shows an expanded view of part of a trace obtained from the oscilloscope monitoring the probe intensity following a pump pulse. The trace corresponds to a length of the fiber incorporating the section contained in the first oven, and this section is responsible for the raised signal level. The spatial resolution, as determined from the rise time of this signal, is approximately $10 \mathrm{~m}$, as expected. To obtain this trace, we averaged the signal 256 times by the oscilloscope.

The potential spatial resolution of the system is, in practice, limited by the Brillouin gain bandwidth, which we measured to be approximately $35 \mathrm{MHz}$ (see, for example, Fig. 4). Reducing the pump pulse period much below 100 ns will result in significant broadening of the Brillouin gain profile with a concomitant worsening of the temperature resolution. In addition, for a given peak pump power, reducing the pulse width results in a decrease in the Brillouin gain and therefore in a reduction in the signal-tonoise ratio. These effects are currently being investigated theoretically.

In conclusion, we have described a DTS system with a sensor length of $22 \mathrm{~km}$ that is able to demonstrate a temperature resolution of $1^{\circ} \mathrm{C}$ and a spatial resolution of $10 \mathrm{~m}$.

The authors gratefully acknowledge the financial support of the UK Science and Engineering Research Council through the Optoelectronics Interdisciplinary Research Centre, Southampton, UK.

\section{References}

1. A. J. Rogers, Electron. Lett. 16, 489 (1980).

2. A. H. Hartog, IEEE J. Lightwave Technol. LT-1, 498 (1983).

3. J. P. Dakin, D. J. Pratt, G. W. Bibby, and J. N. Ross, Electron. Lett. 21, 569 (1985).

4. D. Culverhouse, F. Farahi, C. N. Pannell, and D. A. Jackson, Electron. Lett. 25, 914 (1989).

5. T. Kurashima, T. Horiguchi, and M. Tateda, Opt. Lett. 15, 1038 (1990).

6. See, for example, R. W. Boyd, Nonlinear Optics (Academic, London, 1992), Chap. 8. 